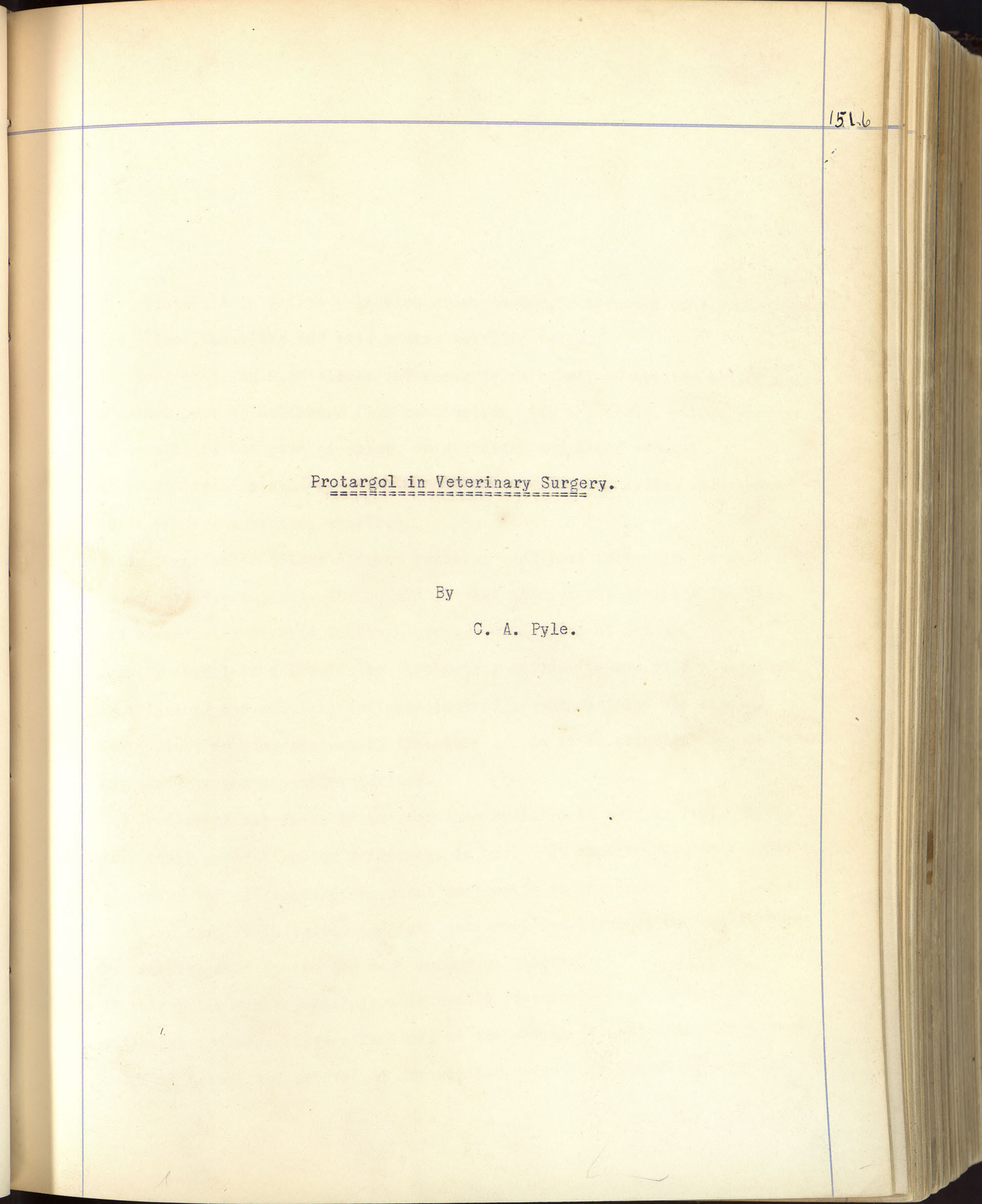


Protargol is a fine yellowish brown powder, a compound of albumen and silver, odorless and with a weak metallic taste. The drug contains $8.3 \%$ silver and seems to be a salt containing silver in a masked form as well as a firm combination with a proteid. This product is soluble in one part of water, in glycerine and blood serum. It is not precipitated by alkaline sulphates, albumen, sodium chloride or acids and has a neutral reaction.

It is superior to Silver Nitrate in being much less irritating, indifferent to sodium chloride and albumen and in that it does not discolor the skin and exceeds the Nitrate in certainty and efficiency of action.

Protargol is a powerfully penetrating antiseptic and mild astringent in catarrhal and purulent inflammations of mucous cavities and tracts. In purulent wounds, abscesses, fistulous tracts it is effective in destroying bacteria and promoting healing.

Protargol was first introduced into medicine in 1897 by Prof. Neisser, as a local medication for gonorrhoea in man. It is also used as a local treatment for catarrhal or purulent urethritis in the Human.

The drug is indicated in acute catarrhal and purulent conjunctivitis in from one half to ten per cent aquous solutions. In tubercular ulcers, fistulous tracts it is reported very effectual. In catarrhel and une retive affections of the ear it is indicated. Abscesses, purulent arthritis, persistant granulating wounds and wounds of the foot 
yield readily to this drug.

Internally the drug is indicated in canine practice as a relief of hemorrhages and ulcerations of the alimentary tract and purulent inflammations of the qenito urinary tract.

The drug in powder or in solution must be preserved from the action of light or heat. In making the solutions the menstruum should be cold and the drug placed on the surface and allowed to dissolve slowly or with gentle agitation. The solution is reddish brown in color depending on the strength of the drug.

\section{ORIGINAL WORK.}

CASE NO. I. A sorrel filly two years old owned by Mr. H. of Randolph, Kansas. At the anterior part of the base of the conchal cartilage of left ear, a small swelling with an opening on superior part discharging an odorless, glairy material in small amount. Colt sensitive toward manipulation of the ear.

History- Cause unknown. Owner reported the condition as present for a year and that it had resisted his efforts at treatment with liniments, lunar caustic, ete.

TREATMENT. Mar. 2.

Restrained the colt with a twitch and laid open the swelling after cleaning off the discharge and trimming the hair. Washed the surface and advised the owner to dress the ear by washing with carbolized water. 
Mar. 9.

Colt doing nicely, swelling somewhat reduced. Procured a small quantity of Protargol and advised its use in solution of about $10 \mathrm{gr}$. to a tablespoonful of water injected after cleansing the wound with carbolized water.

Mar. 16.

Healing was proceeding too rapidly on the outside. Owner was simply injecting the solution thru a small opening kept open by the use of a nail. I again laid open the abscess and made a free incision to keep the wound well open. At the base of the cavity was a small quantity of rather inspissated pus. Advisad continuation of the treatment hoping to induce encapsulation.

Mar. 23.

Region about the wound not so tender, discharge less. Owner thot that it was doing nicely but I considered that the healing at the deeper part was not satisfactory. Advised continuation of the treatment. Expeet to operate for dentigerous cyst at next visit. Apr. 13.

Cast the patient and operated for dentigerous cyst. Removed two small teeth. Dressed as open wound and advised the use of protargol as before.

Apr. 22. Patient reported as nearly healed.

May 20. Owner reports the wound as closed with no discharge. 
CASE NO. 2 .

POLL-EEVIL.

Bay Mare about 12 years old, pregnant, in fair condition, considerable swelling on poll especially on the right side, scars in two or three places, a small opening near line of mane eight inches from occipital crest. Very little discharge, not painful. No history given. TREATMENT. Began Nar. 16.

Placed the mare in the stocks, restrained with a twitch. Probed the opening and with a probe pointed curved bistoury laid open the fistulous' tract to get drainage. In so doing I cut thru a mass of dense fibrous tissue which seemed to replace partly the Obliquitous Capitus Posticus muscle. Considerable hemorrhase and the wound was packed with oakum and four interrupted sutures inserted.

Mar. 16.

Removed the sutures and washed with cold water. Mar. 17.

Washed the wound with warm water. Mixed Protargol $\ddot{y}$. with glycerine $80 \mathrm{c.c}$. and aqua $100 \mathrm{c.c}$. Injected a half ounce of the mixture into the wound and packed with cotton. Applied with rubbing a cantharides blister to each side of the Poll. Mar. 18-to 23 .

Treatment continued daily. Washed the wound with cold water. Washed the discharge from neck and applied adeps to protect the skin and hair. Abundant discharge so reduced the solution to protargol $\bar{j}$ to 
glycerine $80 \mathrm{c.c}$. and aqua $100 \mathrm{c.c}$. This proved more mild. Injected each day one half ounce of the solution and inserted a piece of cotton to hold the material in the cavity.

Mar. 24.

Washed and dressed the wound as usual. Enlarged the opening by an incission toward the poll to allow better application of the treatment. Found that the superior part of Dentata and inferior part of the ligamentum nucha necrosed and quite a large cavity in the region of the poll. Patient keeps up we11, looks healthy. Wound in healthy condition. Not the pain and depression such cases usually suffer. Mar. 25-30.

Treatment same. Considerable discharge from the wound. Mar. 31- Apr. 7.

Dressed the wound as usual but changed the solution to one of $2 \%$ and injected a syringeful into the cavity holding it there with a quantity of cotion. Discharge about the same. Nore odor. Apr. $8-15$.

Dressed the wound as usual using a $5 \%$ solution of Protargol. More discharge. Wound does not look so healthy and drainage not good. Apr. 16.

Opened a soft enlargement on the top of poll over the ligamentum quchae and washed out a quantity of pus. Fnlarged opening to communicatè with the main cavity and dressed as usual. 
Apr. $17-24$

Washed and dressed the patient's head as usual, injecting 4 per cent solution protargol. Mare is due to foal in a few days and is not in good dondition for the wound to heal. Advise deferring further treatment 'till. after parturition when more surgical interference will be permissible. May. 4.

Mare has foaled. Owner will take the mare home to care for the colt. Treatment deferred.

CASE NO. 3. EISTULA:

Brown stallion about $3 \mathrm{yrs}$. old, very poor in condition, ill kept. lousy. History vague, evidently a chronic case-had been treated by a local veterinarian for some time. Depressions and cicatrices on withers indicate that trouble there has been treated but a fistula on the neck about the first dorsal vertebra remains and discharges a small quantity of offensive pus. Probe introduced passes seven or eight inches downward and inward.

TREATVENT began Mar. 28 .

Placed the patient in the stocks, restrained with twitch and laid open the skin and muscles to the bottom of fistulous dract to get drainage, controlled hemorrhage, packed with oakum and inserted two stitches to hold in place. 
$\operatorname{Mar} .29$.

Dressed the wound with cold water and injected a syringe full of protar. gol solution, 4 per cent, and packed with cotton to hold it in place in the wound.

Colt is lame and weak. Requires careful feeding and grooming. Needs assistance to get up when down.

Mar. 30-31.

Dressed the wound as on previous day. Curried thoroly and advised feeding a little oil meal to stimulate the skin. Alkano is being given daily as a tonic.

Apr. $1-8$

Washed the wound with cold water and injected $2 \%$ solution protarsol The tissues seem to separate and allow the pus to burrow. Drainage not good. Patient seems stronger and more active.

Apr. $9-16$.

Made an opening thru Levator Humeri and skin from the bottom of the wound using a large seton needle and enlarging the opening to get drainage. Dressed as usual using a 5 per cent solution of protargol. Colt is gaining in flesh and strength. The wound looks healthy. Apr. 16-24.

Washed and treated the patient as usual but omitted the arug. Apr. 25.

Owner wishes to take the colt home and treat it himself. He was given 
a week's supply of Protargol with instructions to apply it as usual and expected to return it at intervals for examinations and further treatment. Nothing of the development has been reported and evidently the treatment was stopped.

CASE NO. 4. CATARRHAL CONJUNCTIVITIS.

Bay Nare 9 yrs. old. Owner A. W. Carlson, Randolph, Kansas. History. Mare had good conformatian, strong eyes but about a month previous the left eye became badly inflamed, sensitive and with abundant lachrymal discharge. Owner knew of no recent trauma and of no trouble during the past two years. The left eye was fairly cleat, but conjunctiva swollen red and inflamed. Right eye clear and about normal.

TREATMENT. MaY 14.

Ordered the druggist to supply protargol 36 grains to two ounces of water. Instructed using a curved pointed medicine dropper to instill the small syringe full into the eve three times daily. May 21.

Eye cleared up pretty well, laebrymation not excessive, conjunctiva nearly normal. Recovery.

These cases being 211 that the clinic afforded for original work, the reports of experimental and practical uses of the drug in other hands would be useful in demonstrating the use of the salt in veterinary medicine. 
Dr. W. E. A. Wyman in 1901 used the drug in quite an extensive practice being supplied by the manufacturers free of cost, and reports success in treating the following eases.

1. Empyema of the nasal sinuses after trephining and expulsion of tooth. Treatment. Injections two or three times daily of a 5 per cent solution of Protargol. Discharge stopped on an average in 16 days.

2. Fistulous withers and Poll Evil.

Treatment. Surgical interference to get drainage and remove necrosed tissue. Irrigation with one half per cent formalin or 2 per cent creolin solutions and injections of Protargol ten per cent, glycerine fifty per cent, and aqua forty per cent twice daily.

Recovery in 2 to 5 weeks.

3. Otitis Extema (Dog)

Treatment. Cleanse with warm alkaline solution, drying and injecting Protargol 20 per cent, One and one half drachms, into the ear duct twice daily. Curettement sometimes necessary.

4. Dermatitis Eczematosa (Scratches d)

Treatment. Wash the foot and limb in warm water and soan, dry and apply Protargol 10-20 per cent in adeps or Tanolin. Apply by rubbing the ointment on a eloth and secure with a bandage.

Effective in $10-14$ days.

5. Punctured infected wound in front of Patella caused by a pitch fork. Treatment. Openings enlarged and bubbled out with $\mathrm{H}_{2} \mathrm{O}_{2}$ and 
protargol solution, ten per cent injected every three hours with hot water irrigations.

Recovery in 6 days.

6. Purulent Conjunctivitis.

Treatment. Syringing out the conjunctival sac twice daily with 1-5 per cent solution of protargol.

Recovery in $5-10$ days.

From the Rec. De. Vét. Méd. is translated a record of the use of the drug by Hendieckx in purulent traumatic arthritis of the hock in very grave condition.

Treatment. Injection of an ounce of 3 per cent solution into the joint with constant irrigations.

\section{Rapid recovery.}

Dr. Albrecht of Munich reports the use of the drug with success in the following cases.

1. Otitis Externa in the dog. Four cases. Purulent offensive discharge from the external ear.

Treatment. Cleansed the ear with a sponoge held in a pair of forceps and soaked in a one per cent lysol soultion. Drying and instilling into external ear 1 to 2 c.c. of a 3 per cent protargol solution and closing the meatus by pressing on the ear with the fingers.

In alll cases a recovery after 8 to 10 days treatment. 
2. A fox terrior with preputial catarrh.

Treatment. Injecting $5 \mathrm{c.c}$. of a 3 per cent protarol solution into the preputial cavity and manipulating gently to force the solution to all parts.

\section{Cured in 8 days.}

3. Purulent Opthalmia (Dog) with a large corneal ulcer and a profuse catarrhal discharge.

Treatment. Daily installations of fifteen drops of a 1 per cent protarol solution.

In fourteen days eye had returned to the normal except for a slight cicatricial tissue.

Dr. Roth, a colleague of Prof. Albrecht's reports the following cases.

Otitis Externa. Seven cases at various times in shepherd dogs. Treatment about the same in each case. Daily instillations of fifteen drops of a 3 per cent solution of protargol into the ear and the ear held closed and a plug of cotton inserted.

Recovery in 2 to 5 weeks.

OBSERVATIONS Protargol is quite expensive and so rendered impractical where it must be used in considerable quantity.

There is difficulty in large cavities having good drainage and wounds of considerable extent, in holding the solution to get the desired action. 
A proper mixture of the powdered drug with Boracic Acid,Carboligni and amylum might be more affective and used as a dressing powder. SUMMARY.

1. Protargol ia a powerfully penetrating bactericidal agent.

2. It is non irritating and is especially practical and useful in inflamed conditions of mucous and serous cavities.

3. It is most effective in inflamed and purulent conditions of the eye and ear in dogs.

4. It induces kealthy granulations and cicatrizations.

CONCLUSION. Protargol is a comparatively new remedy with possibly more indications but has a definite, useful place in veterinary surgery. 